\title{
The Comparison Between Surgical Procedure and Conservative Treatment in the Management of Traumatic Subdural Effusion
}

\author{
Dajiang XIE, Jixi XIE, Yingfeng WAN, Xuchen QI, Shuxu YANG \\ Zhejiang University, College of Medical Sciences, Sir Run Run Shaw Hospital, Department of Neurosurgery, Hangzhou, China
}

\section{ABSTRACT}

AIM: Traumatic subdural effusion (TSE) occurs following traumatic brain injury and may be treated by either conservative methods or surgical procedure commonly according to the patients' clinical information. We aimed to compare the effective rate of effusion removal and the standardized morbidity ratio of poor prognosis of the two different treatments, and to discuss the future treatment methods possible.

MATERIAL and METHODS: We reviewed the clinical records of patients who were divided into two groups according to the treatment choices in our center, and the effective rate of effusion removal and the standardized morbidity ratio of poor prognosis were compared.

RESULTS: Eighty patients were identified, and divided into two groups: conservative treatment and surgical procedure group. The mean CRASH-CT predicted risk of mortality in two weeks and unfavorable outcome at six months was higher in the surgical procedure group compared with the conservative treatment group.

Effective rate of effusion removal was observed in $57.1 \%$ of conservative treatment group versus $88.5 \%$ of surgical procedure group ( $\mathrm{p}=0.002)$. The standardized morbidity ratio of poor prognosis (observed/expected poor prognosis) was 0.56 (95\% $\mathrm{Cl}$ : 0.32-0.80) for the conservative treatment group versus 0.25 (95\% Cl: 18 0.08-0.42) for the surgical procedure group.

CONCLUSION: Conservative treatment and surgical procedure are used for the management of traumatic subdural effusion, and the former is used more commonly to treat the mildly affected patients than the latter one, but a surgical procedure may be more effective for the patients in poor clinical condition. Adequate evidence is required to clear the indications.

KEYWORDS: Traumatic subdural effusion, Conservative treatment, Surgical procedure

Abbreviations: TSE: Traumatic subdural effusion, CTG: Conservative treatment group, SPG: Surgical procedure group, CRASH: Corticoid randomisation after significant head injury, CT: Computed tomography, CSF: Cerebrospinal fluid, GOS: Glasgow outcome scale, STROBE: Strengthening the reporting of observational studies in epidemiology.

\section{INTRODUCTION}

$\mathrm{T}$ raumatic subdural effusion is a common disease, and has a high percentage in traumatic head injury cases $(2,4,7,12,19,20)$. Traumatic subdural effusion occurs as the accumulation of cerebrospinal fluid (CSF) after the traumatic arachnoid rupture $(2,11,20)$. Poor prognosis appears when mass effect, serious brain injury or intracranial hematoma occur, and the mortality is high $(1,2,4)$. 
Traumatic subdural effusion is classified into acute, subacute, and chronic types according to the interval between injury and the appearance of clinical symptoms (1). It also can be classified into four types, such as resolution, steadiness, development and evolution type according to dynamic findings of computed tomographic scan $(11,13)$.

Though many investigators suggest that conservative treatment is the first choice, a surgical procedure is necessary when the mass effect and unfavorable clinical manifestations occur $(2,5)$. Surgery should be performed for most patients with a development or evolution type $(2,11)$. The options of surgical procedures included drilling drainage, subduralperitoneal shunt and hematoma or hygroma evacuation $(2,9)$. External drainage is the first surgical option because of the low infection rate $(2,10)$. However, the effusion may recollect in a certain proportion after drilling drainage, which can be prevented by the shunt procedure. Evacuation is necessary when the effusion forms a cyst. The objective of this paper is to compare the outcomes following the two treatment, the observed with predicted clinical outcome, find out the significant differences, review the current literature and discuss future treatment methods.

\section{- MATERIAL and METHODS}

This article is a retrospective study of the past clinical data. We collected all the cases after traumatic subdural effusion without obvious brain parenchymal damages. Eighty patients (40 females and 40 males) with a mean age of 55 years (ranging from 41 to 70 years) from July 2006 to August 2013 were identified from 158 patients with subdural effusion, and 158 patients were entered as 'subdural effusion' and 'surgical procedure' or 'conservative treatment' in our center (Figure 1). Diagnostic criteria of traumatic subdural effusion are as follows (Figure 2A-D) (6):

a. Hydroma, appearing within 10 days after trauma.

b. Computed tomography (CT) scan showing a crescentshaped homogenous low-density area similar to CSF, with compression of the adjacent portions of the cerebrum, and effacement of adjacent sulci.

c. CT scan value of the lesion is below $20 \mathrm{Hu}$.

d. The membrane of lesion is usually not enhanced.

Exclusion criteria were: non-traumatic cases of subdural effusion, subdural effusion accompanies with apparent parenchymal brain injury, and intracranial hematoma.

The choice of treatment technique depended on the neurosurgeons' judgment to clinical symptoms and signs. As a general rule, surgery should be performed on the basis of the clinical presentations (Brain compression symptoms or epileptic seizure) and CT scan characteristics (supratentorial effusion volume $>25 \mathrm{ml}$, infratentorial effusion volume $>8 \mathrm{ml}$; obvious mass effect, increased intracranial pressure, midline shift $>10 \mathrm{~mm}$ ). In the conservative group, the consciousness and blood electrolytes of patients were closely detected, prevention of epilepsy and neurotrophic therapy were performed. Optional sur- gical methods which can remove the effusion included drilling drainage, subdural peritoneal shunt, hygroma or hematoma evacuation. Shunt surgery played an important role when the drilling drainage failed to reduce effusion volume or lead to worse clinical symptoms.

Predicted outcome was evaluated using the CRASH head injury prediction model when the patients were admitted to our center (3). It was proven that the model is available for outcome prediction in traumatic brain injury on the basis of admission condition and computed tomographic scan data $(13,14)$. The risks of 2 -week mortality and poor outcome at six months (Glasgow Outcome Score $\leq 3$ ) were calculated for each patient and the mean risk was calculated for each group separately (Figure 3). The standardized morbidity ratio of unfavorable outcome at six months was calculated as the ratio of observed against predicted count of unfavorable events.

Effective rate of effusion removal (Complete obliteration $(100 \%)$, significant removal $(\geq 75 \%)$ and moderate removal (25\%-75\%)) was calculated for each group. The postoperative condition was evaluated using the Glasgow Outcome Scale system (GOS) and classified into five groups such as good recovery (stage 1), moderate disability (stage 2), severe disability (stage 3), persistent vegetative state (stage 4), and death (stage 5).

Continuous variables were compared using the unpaired Students't-test or the Mann-Whitney test and categorical variables were compared using the Fisher's exact test or, if more than two possible categories, using the chi squared test. The $95 \% \mathrm{Cl}$ for the standardized morbidity ratio was the confidence interval at $95 \%$. This study conforms to the STROBE statement (18).

\section{RESULTS}

Eighty patients of explicit traumatic subdural effusion underwent the two different management methods after a professional judgment process and obtaining informed consent (Table I). Forty-two (52.5\%) of the patients were in the conservative treatment group and thirty-eight $(47.5 \%)$ of the patients were in surgical procedure group (Figure 2A-D). The median age was 53 years and $50 \%$ of the patients were male. The Glasgow Coma Score (GCS) of admission was $\leq 8$ in $6(7.5 \%)$ patients, $9-12$ in $34(42.5 \%)$ patients and $\geq 13$ in $40(50 \%)$ patients. All the admission data, including computed tomographic scan, were available for the predicted mortality at 14 days and death or severe disability at six months.

The surgical procedure group was significantly older (median age 60 years in the surgical procedure group versus 50 years in the conservative treatment group, $p=0.032$ ). It had a larger composition of patients presenting with unfavorable pupillary reaction (47.4\% in the surgical procedure group versus $2.4 \%$ in the conservative treatment group, $p=0.001$ ) and Glasgow Coma Score $\leq 8(15.8 \%$ in the surgical procedure group versus 0 in the conservative treatment group, $p=0.007)$. Data on computed tomographic scan have significant differences, including the presence of obliteration of the third ventricle or basal cisterns $39.5 \%$ in the surgical procedure group versus 

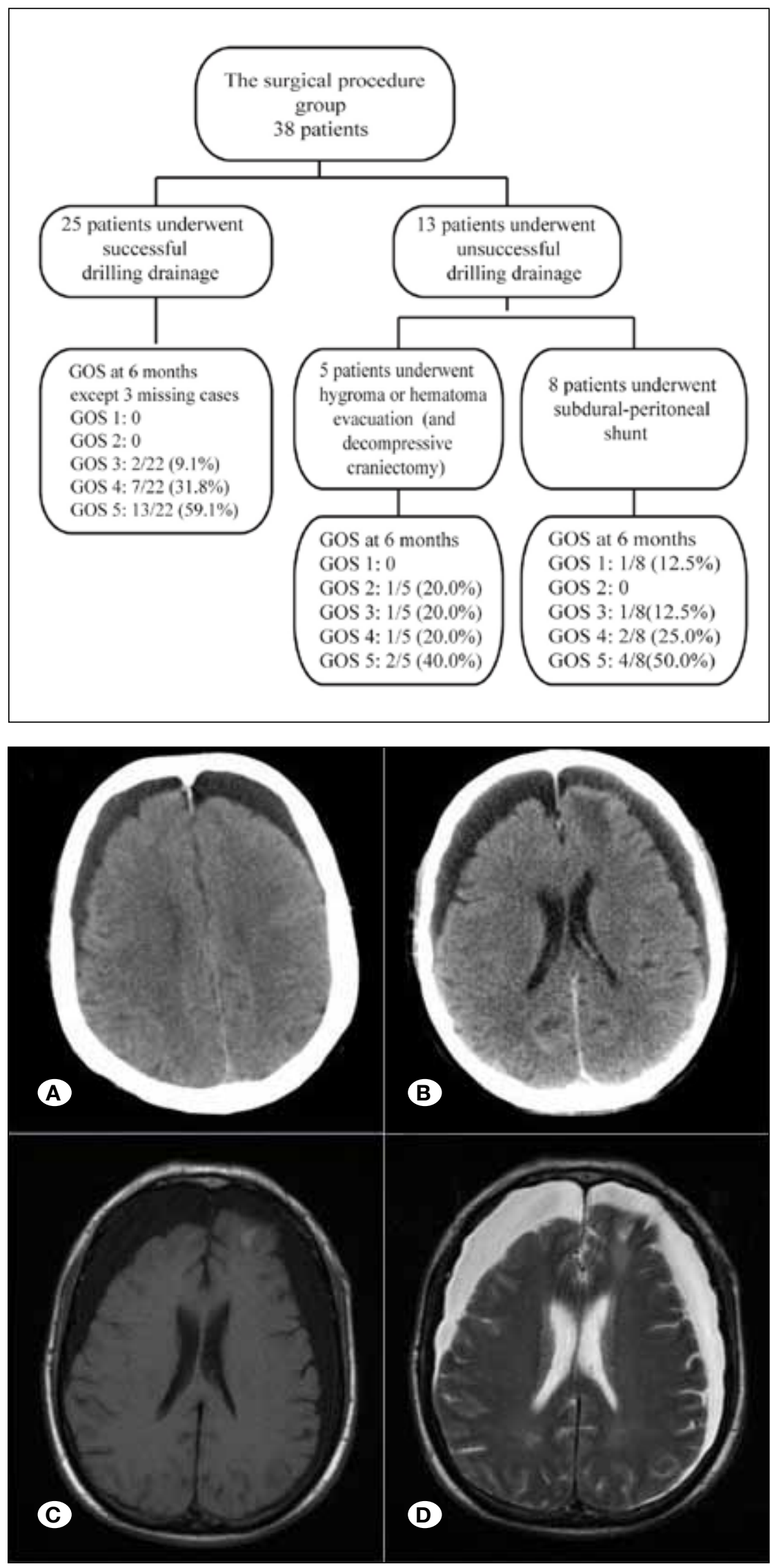

Figure 1: Flowchart and outcome of the surgical procedure group.
Figure 2: Imaging examination of subdural effusion A, B) axial computed tomography scan show a crescent-shaped homogenous lowdensity area. C, D) axial magnetic resonance imaging scan demonstrate a well-defined T1-hypointense (C) and T2-hyperintense (D) homogeneous subdural lesion. 
$16.7 \%$ in the conservative treatment group, $p=0.023$ ), and the midline shift $(100 \%$ in the surgical procedure group versus $47.2 \%$ in the conservative treatment group, $p=0.001$ ).

A significant difference existed in the predicted risks of death at 14 days and unfavorable outcome at six months between the conservation group and the surgical procedure group. The mean predicted risk of death at 14 days for the conservative group was 16.7\% (95\% Cl: 5.4-28.0) versus $50.0 \%$ (95\% Cl: 34.1-65.9) for the surgical procedure group, and the predicted risk of unfavorable outcome at six months for the conservative group was $40.5 \%$ (95 \% Cl: $25.7-55.3)$ versus $68.6 \%(95 \% \mathrm{Cl}$ : 53.2-84.0) for the surgical procedure group (Figure 3). These data suggested that the admission condition of patients who underwent surgical procedure were more serious, and the risk of unfavorable outcome (death, persistent vegetative state, severe disability) at six months was more than the risk of death at two weeks.

GOS at six months was available for all of the patients in the conservative treatment group and 35 patients in the surgical procedure group. The mortality rate was $4.8 \%$ (2 patients) in the consersative group and $2.9 \%$ (2 patients) in the surgical procedure group $(p=0.667)$. There were 9 patients $(21.4 \%)$ of unfavorable outcomes (death, persistent vegetative state, severe disability) in the conservative group and 33 patients

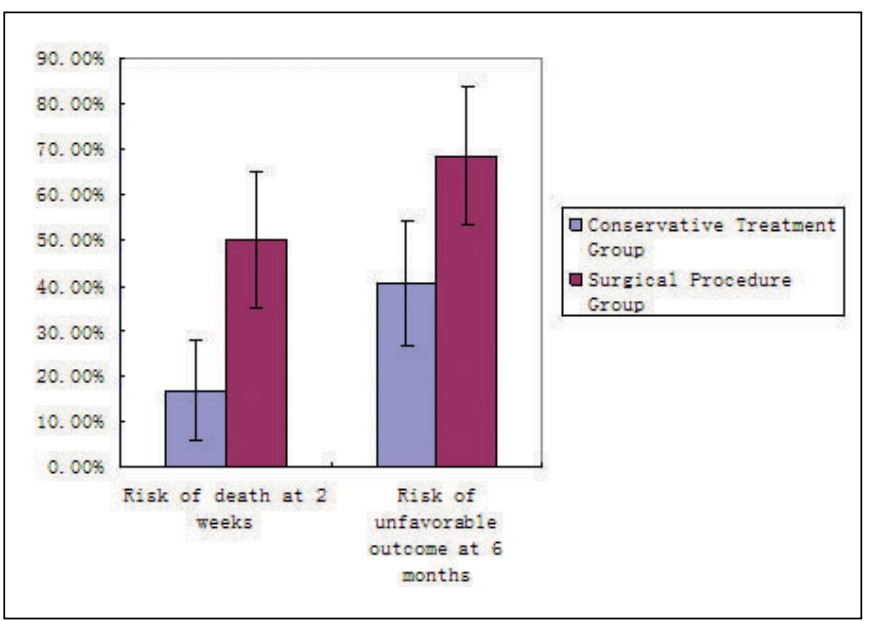

Figure 3: The risk of death at 2 weeks and unfavorable outcome at six months.

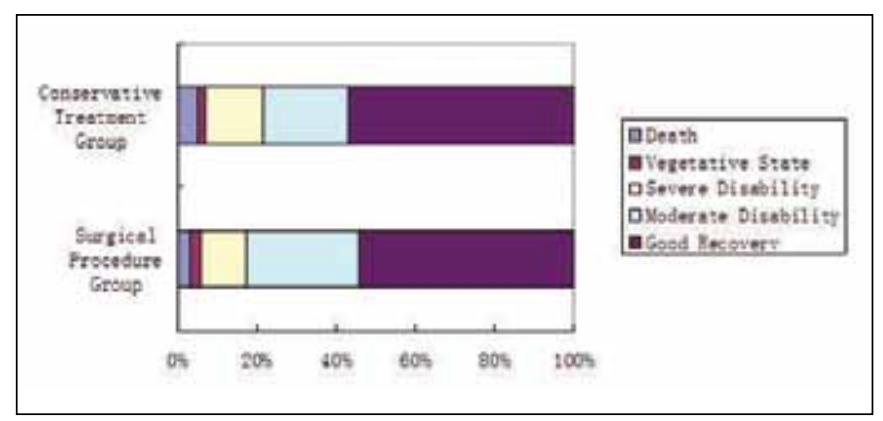

Figure 4: Glasgow Outcome Scale scores between the two treatment groups at six months.
(78.6\%) of favorable outcomes (moderate disability, good recovery). There were 6 patients $(17.1 \%)$ with unfavorable outcomes (death, persistent vegetative state, severe disability) in the surgical procedure group and 29 patients (82.9\%) with favorable outcomes (moderate disability, good recovery). A significant difference of the two outcomes did not exist between the two groups ( $p=0.636$; Figure 4$)$. Effective rate of effusion removal was observed in $57.1 \%$ of conservative treatment group versus $88.5 \%$ of surgical procedure group $(p=0.002$; Table II). The disability rate was $38.1 \%$ (16 patients) in the conversation group versus $42.9 \%$ (15 patients) in the surgical procedure group $(p=0.671)$, and the standardized morbidity ratio of unfavorable outcome was $0.56(95 \% \mathrm{Cl}$ : $0.32-0.80$ ) for the conservative treatment group versus 0.25 (95\% Cl: 0.08-0.42) for the surgical procedure group (Figure $5)$.

\section{DISCUSSION}

Subdural effusion was described as the accumulation of cerebrospinal fluid in the subdural space for centuries, and the first description is credited to Mayo in $1893(6,11)$. Different causes have been identified including previous surgical procedures (15), accidental head injury, intracranial infection, congenital malformations, and cranio-encephalic disproportion, according to Caldarelli et al. (2). Traumatic subdural effusion which is considered a delayed traumatic lesion is a common lesion following both major and minor head injury in several hours or one week.

The treatment of traumatic subdural effusion is controversial, and there are inconsistent conclusions about the benefit of the surgical procedure. Some investigators indicated that conservative methods is the appropriate one for its spontaneous resolution $(5,8,19)$. Others suggested that surgical procedure is necessary for the complications of mass effect and it can relieve patients' suffering immediately $(2,16)$.

The comparison of the conservative treatment and surgical procedure of subdural effusion in clinical presentation, computed tomographic scan and prognosis had been reported by

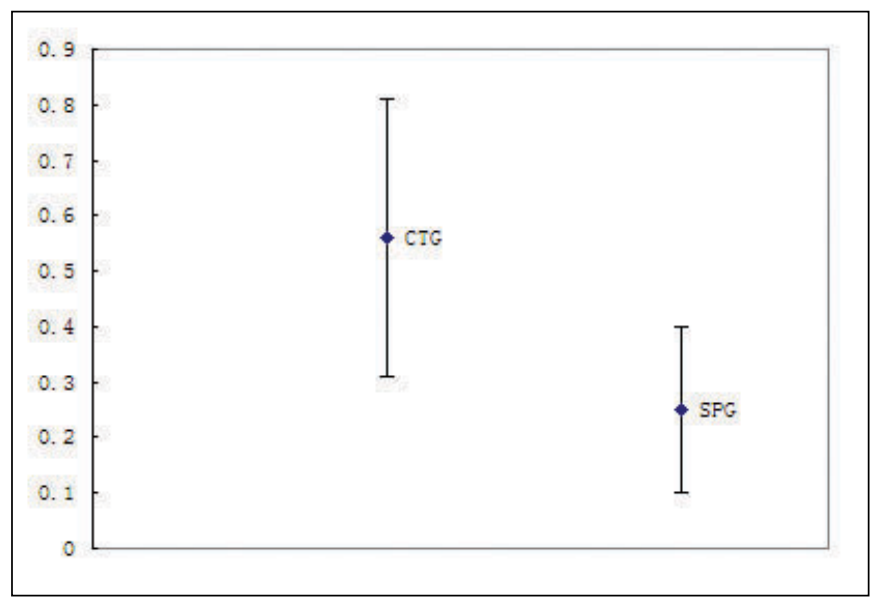

Figure 5: Standardized Morbidity Ratio for the Conservative Treatment Group (CTG) and the Surgical Procedure Group (SPG). 
only few investigators. In the study of Jaccard and de Tribolet, conservative treatment was the most appropriate one, because the surgical treatment of the effusion seldom improved the patients' condition according to the clinical presentation and computed tomographic scan (5).

John and his colleagues indicated that there was marked diversity in the severity of subdural effusion (6). The lesion was described as remained; reduced, resolved, enlarged and changed, subdural effusion can change into subdural hematoma as a precursor at 101.5 days in $33 \%$ of the patients on the basis of computed tomographic scan, according to Lee and his colleagues (9). So the treatment choice is controversial. As the effusion usually resolves spontaneously, conservative treatment seems an appropriate method, according to Jaccard and de Tribolet (5), but subdural effusion can produce increased intracranial pressure (ICP) and/or focal neurological deficits resulting from local compression on brain parenchyma, favorable outcomes can be obtained via the aggressive surgical procedure $(2,16)$.

We undertook this study because it would be important for those treating patients with subdural effusion to know if surgical procedure is more effective than conservative

Table I: Admission Data for the Patients for whom both Baseline Predictive Data and Outcomes at 6 Months were Available IQR Stands for the $25 \%-75 \%$ Interquartile Range

\begin{tabular}{|c|c|c|c|c|}
\hline & & $\begin{array}{l}\text { The conservative } \\
\text { treatment group }\end{array}$ & $\begin{array}{l}\text { The surgical } \\
\text { procedure group }\end{array}$ & \\
\hline Number of cases & & $42(52.5 \%)$ & $38(47.5 \%)$ & \\
\hline Age & & 50 years & 60 years & $\mathrm{P}=0.032^{*}$ \\
\hline Gender & & $\begin{array}{l}22 \text { male }(52 \%) \\
20 \text { female }(48 \%)\end{array}$ & $\begin{array}{c}18 \text { male }(47 \%) \\
20 \text { female }(53 \%)\end{array}$ & $P=0.654$ \\
\hline Consciousness & $\begin{array}{l}\text { Clear consciousness } \\
\text { Disturbed consciousness }\end{array}$ & $\begin{array}{l}40(95.2 \%) \\
2(4.8 \%)\end{array}$ & $\begin{array}{l}16(42.1 \%) \\
22(57.9 \%)\end{array}$ & $P=0.001^{*}$ \\
\hline Pupils & $\begin{array}{l}\text { Both reactive } \\
\text { One reactive } \\
\text { None reactive }\end{array}$ & $\begin{array}{c}41(97.6 \%) \\
1(2.4 \%) \\
0 \\
\end{array}$ & $\begin{array}{c}20(52.6 \%) \\
16(42.2 \%) \\
2(5.2 \%) \\
\end{array}$ & $P=0.001^{*}$ \\
\hline Muscle weakness & $\begin{array}{l}\text { Both sides } \\
\text { One side } \\
\text { None side }\end{array}$ & $\begin{array}{c}8(19.1 \%) \\
19(45.2 \%) \\
15(35.7 \%) \\
\end{array}$ & $\begin{array}{c}25(65.8 \%) \\
10(26.3 \%) \\
3(7.9 \%) \\
\end{array}$ & $P=0.001^{*}$ \\
\hline GCS scores & $\begin{array}{l}\geq 13 \\
9-12 \\
\leq 8\end{array}$ & $\begin{array}{c}38(90.5 \%) \\
4(9.5 \%) \\
0(0 \%)\end{array}$ & $\begin{array}{c}2(5.3 \%) \\
30(78.9 \%) \\
6(15.8 \%)\end{array}$ & $P=0.001^{*}$ \\
\hline Psychiatric symptoms & & $4(9.5 \%)$ & $7(18.4 \%)$ & $P=0.249$ \\
\hline CT scan & $\begin{array}{l}\text { Median volume of effusion (ml) } \\
\text { Obstructed path of CSF } \\
\text { circulation } \\
\text { Midline Shift } \\
\text { Median midline shift (mm) } \\
\text { Encephalatrophy }\end{array}$ & $\begin{array}{c}18 \text { (Q1-Q3: 8-20) } \\
7(16.7 \%) \\
\\
20(47.2 \%) \\
8(\mathrm{Q} 1-\mathrm{Q} 3: 5-10) \\
4(9.5 \%)\end{array}$ & $\begin{array}{c}45 \text { (Q1-Q3: 25-60) } \\
15(39.5 \%) \\
\\
38(100 \%) \\
12 \text { (Q1-Q3: 8-17) } \\
7(18.4 \%)\end{array}$ & $P=0.001^{*}$ \\
\hline Underlying diseases & & $10(23.8 \%)$ & 15 (39.5\%) & $P=0.132$ \\
\hline
\end{tabular}

*Statistically significant.

Table II: Change of Effusion After Six Months

\begin{tabular}{lccccccc}
\hline & $\begin{array}{c}\text { Complete } \\
\text { obliteration }\end{array}$ & $\begin{array}{c}\text { Significant } \\
\text { reduction }\end{array}$ & $\begin{array}{c}\text { Moderate } \\
\text { reduction }\end{array}$ & $\begin{array}{c}\text { Mild } \\
\text { reduction }\end{array}$ & $\begin{array}{c}\text { Unchanged } \\
\text { volume }\end{array}$ & Recurrence & Evolution \\
\hline Conservative & $8 / 42$ & $9 / 42$ & $7 / 42$ & $5 / 42$ & $9 / 42$ & $1 / 42$ & $3 / 42$ \\
treatment group & $(19.0 \%)$ & $(21.4 \%)$ & $(16.7 \%)$ & $(11.9 \%)$ & $(21.4 \%)$ & $(2.4 \%)$ & $(7.2 \%)$ \\
\hline \multirow{2}{*}{ Surgical procedure group } & $15 / 35$ & $10 / 35$ & $6 / 35$ & $2 / 35$ & & $1 / 35$ & $1 / 35$ \\
& $(42.8 \%)$ & $(28.6 \%)$ & $(17.1 \%)$ & $(5.7 \%)$ & 0 & $(2.9 \%)$ & $(2.9 \%)$ \\
\hline
\end{tabular}


treatment. There are two differences in our study from the former studies: 1) The patients were divided into two groups on basis of their conditions; and 2) the clinical data were collected and calculated at different times. The design is to explore the question of differences between the methods in the treatment of the subdural effusion.

The results show that two methods can obtain favorable outcomes, were favorable in the treatment of the subdural effusion, and significant difference of the outcomes did not exist between the two groups. The surgical procedure group had a higher effective rate of effusion removal and lower standardized morbidity ratio of unfavorable outcome.

A number of studies have shown that the outcome in patients with subdural effusion is related to primary injury and admission data, such as GCS, age, papillary response, instead of the effusion itself $(5,9)$. Other factors are important in determining outcome. Tsuang et al. indicated that concomitant hydrocephalus often affect the outcome (17).

Selection bias existed in our study. Surgeons chose the severely injured patients who met the surgical indications to undergo surgical procedure, in anticipation of remission of mass effect and complications. Compared with the conservative treatment, surgical wound played an important role.

The medicines were likely to play an important role in the outcome. Prophylactic intravenous antibiotic therapy was routinely applied in all patients after surgical procedure, so it was beneficial to prevent potential infection. In the conservative group, antibiotics were used only for explicit infection, so the patients in poor condition may be exposed to a different infection risk.

We believe that the absence of outcome differences despite a higher predicted risk for mortality and unfavorable outcome and lower standardized morbidity ratio of unfavorable outcome in the surgical procedure group possibly suggests that primary surgical procedure may be more effective than conservative treatment for selected patients.

Complications of the two methods exist in the therapeutic process. Conservative treatment may have the risk of serious conditions occurring. Surgical complications, such as hemorrhage, encephaledema, infection, leakage of cerebrospinal fluid, are possible. Excessive shunt and blocking of tube are the special complications of subdural-peritoneal shunt.

It is therefore likely that in special condition one treatment can have a beneficial effect compared with the other, conservative treatment is fit for the patients with no or milder clinical presentation, and the patients with plenty of effusion, explicit mass effect and other severe brain injuries need a surgical procedure. Surgical treatment can remove effusion, capsule and hematoma to improve the clinical presentation.

Nevertheless, the key issue is that there is no explicit evidence with retrospective studies exploring the real role of two methods in the management of subdural effusion. We expect a randomized trial with more precise inclusion and exclusion criteria and endpoints to resolve the unanswered questions.
Our study has its own limitations. First, the clinical data were collected from a single centre. Second, we made a necessary assumption that the algorithm of CRASH model and predicted outcome was universal. Third, it is a retrospective study which limited time of follow-up and the rate of loss to follow-up existed.

\section{- CONCLUSIONS}

The two methods are favorable in the treatment of the subdural effusion. Conservative treatment and surgical procedure are used for the management of traumatic subdural effusion, and the former is used more commonly to treat the slight patients than the latter one, but surgical procedure may be more effective for the patients in poor clinical condition for its effective rate. However, explicit indications of conservative treatment and surgical procedure remain a matter of debate. A randomized trial comparing surgical procedure with conservative treatment of subdural effusion is justified and required.

\section{ACKNOWLEDGEMENT}

This work was supported by Natural Science Foundation of Zhejiang Province of China (Y13H160006).

\section{REFERENCES}

1. Borzone M, Capuzzo T, Perria C, Rivano C, Tercero E: Traumatic subdural hygromas: A report of 70 surgically treated cases. J Neurosurg Sci 27(3):161-165, 1983

2. Caldarelli $M$, Dirocco $C$, Romani R: Surgical treatment of chronic subdural hygromas in infants and children. Acta Neurochir 144(6):581-588,2002

3. Collaborators CT. Available at: http://www.crash.Ishtm.ac.uk/ Risk\%20calculator/index.html Accessed November 24, 2010

4. Herold TJ1, Taylor S, Abbrescia K, Hunter C: Post-traumatic subdural hygroma: Case report. J Emerg Med 27(4):361-366, 2004

5. Jaccard E, de Tribolet N: Post-traumatic subdural hygroma. Neurochirurgie 29(5):333-338,1983

6. John JN, Dila C: Traumatic subdural hydroma in adults. Neurosurgery 9:621-625, 1981

7. Kumar R, Singhal N, Mahapatra AK: Traumatic subdural effusions in children following minor head injury. Childs Nerv Syst 24:1391-1396, 2008

8. Lee KS: The pathogenesis and clinical significance of traumatic subdural hygroma. Brain Inj 12:595-603, 1998

9. Lee KS, Bae WK, Gun H, Yun IG: The fate of traumatic subdural hygroma in serial computed tomographic scans. J Korean Med Sci 15:560-568, 2000

10. Litofsky NS, Raffel C, Mc Comb JG: Management of symptomatic chronic extra-axial fluid collections in pediatric patients. Neurosurgery 21(3):445-450, 1992

11. Liu Y, Gong J, Li F, Wang H, Zhu S, Wu C: Traumatic subdural hydroma: Clinical characteristics and classification. Injury 40: 968-972, 2009 
12. Masuzawa T, Kumagai $M$, Sato F: Computed tomographic evolution of post-traumatic subdural hygroma in young adults. Neuroradiology 26(3):245-248, 1984

13. Murata K: Chronic subdural hematoma may be preceded by persistent traumatic subdural effusion. Neuros Med Chir 33: 691-696, 1993

14. Perel P, Arango M, Clayton T, Edwards P, Komolafe E, Poccock $S$ : Predicting outcome after traumatic brain injury: Practical prognostic models based on large cohort of international patients. BMJ 336:425-429, 2008

15. Sonmez OF, Temel Y, Visser-Vandewalle V, Sahin B, Odacı E: A new evaluation method for the intracranial volume changes and subdural effusion of patients following endoscopic third ventriculostomy. Clin Neurol Neurosurg 115:160-164, 2013

16. Todorow S, Happe M: Traumatic subdural hygromas. Neurochirurgia (Stuttg) 24(4):131-136, 1981
17. Tsuang FY, Huang APH, Tsai YH, Chen JY, Lee JE, Tu Y K, Wang KC: Treatment of patients with traumatic subdural effusion and concomitant hydrocephalus. J Neurosurg 116:558-565, 2012

18. von Elm E, Altman DG, Egger M, Pocock SJ, Gotzsche PC, Vandenbroucke JP: The Strengthening the reporting of observational studies in epidemiology (STROBE) statement: Guidelines for reporting observational studies. Lancet 370:1453-1457, 2007

19. Zanini MA, de Lima Resende LA, de Freitas CCM, Yamashita S: Five cases with changed density and spontaneous resolution. Arq Neuropsiquiatr 65(1):68-72, 2007

20. Zanini MA, de Lima Resende LA, de Souza Faleiros AT, Gabarra RC: Traumatic subdural hygromas: Proposed pathogenesis based classification. J Trauma 64:705-713, 2008 